\title{
A metasurface with bidirectional hyperbolic surface modes and position-sensing applications
}

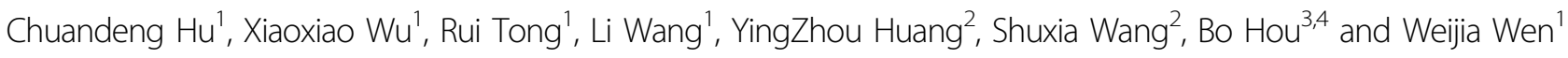

\begin{abstract}
We have theoretically and experimentally studied resonance-induced hyperbolic metasurfaces and proved that they offer an efficient way to introduce Fano-resonance and decrease the Q-factor in our system in order to create hyperbolic isofrequency contours (IFCs) along two orthogonal directions. A metasurface with a continuous topological transition for such IFCs has been designed and experimentally implemented. In particular, two independent collimation frequencies can be found to correspond to the transition frequencies in orthogonal directions. As a consequence, we experimentally demonstrated that the metasurface can function as a position sensor by utilizing bidirectional hyperbolic surface waves, introducing a new avenue for coordinate sensing.
\end{abstract}

\section{Introduction}

Surface electromagnetic (EM) waves on twodimensional interfaces of dissimilar mediums have been a predominant topic of interest among researchers due to their unique features ${ }^{1-3}$. In the optical and near-infrared regime, confined EM waves propagating along the interface between dielectrics and metals are called surface plasmon polaritons (SPPs) and have been widely used in biosensing $^{4}$, superresolution imaging ${ }^{5,6}$, and waveguides ${ }^{7}$. However, metals can be regarded as perfect electric conductors (PECs) in low-frequency ranges, e.g., the farinfrared, microwave, and terahertz regimes. In these lowfrequency regimes, SPPs become very weakly confined Zenneck waves, and the evanescent fields can extend over several wavelengths ${ }^{8,9}$. Fortunately, designer SPPs or spoof SPPs (SSPPs), which mimic the novel properties of SPPs and overcome the aforementioned limits by using structured PECs, were demonstrated in $2004^{10}$.

\footnotetext{
Correspondence: Bo Hou (houbo@suda.edu.cn) or Weijia Wen (phwen@ust.hk) ${ }^{1}$ Department of Physics, The Hong Kong University of Science and Technology, Clear Water Bay, Kowloon 518000 Hong Kong, China

2Department of Applied Physics, Chongqing University, Chongqing 401331, China

Full list of author information is available at the end of the article.

These authors contributed equally: Chuandeng Hu, Xiaoxiao Wu.
}

Metallic/dielectric layers composed of subwavelength, regularly arranged metallic wires known as metasurfaces bind EM fields to individual interfaces with unique anisotropic dispersions that, along with SPPs and SSPPs, play important roles in manipulating near-field light scattering ${ }^{11-14}$.

In addition, hyperbolic metamaterials have attracted substantial research interest due to their special dispersions ${ }^{15-17}$, and research has been extended to the metasurfaces because the in-plane propagating properties are well controlled ${ }^{14,18-20}$. Consequently, special properties such as collimation, which were first studied in photonic crystals, have attracted immense interest in metasurfaces $^{11,18,19,21}$. Such collimating transport exists when the dispersion becomes a flat line, a unique shape between the elliptical and hyperbolic IFCs that has been theoretically studied and experimentally measured at microwave frequencies along a single direction ${ }^{19}$. The gradual change in dispersion line shape leads to a topological transition and confines the propagation direction of SSPPs, which act as Dyakonov-like waves ${ }^{22,23}$. However, this unique collimation exists only in one direction at a single frequency in the previous literature, which limits its application. 

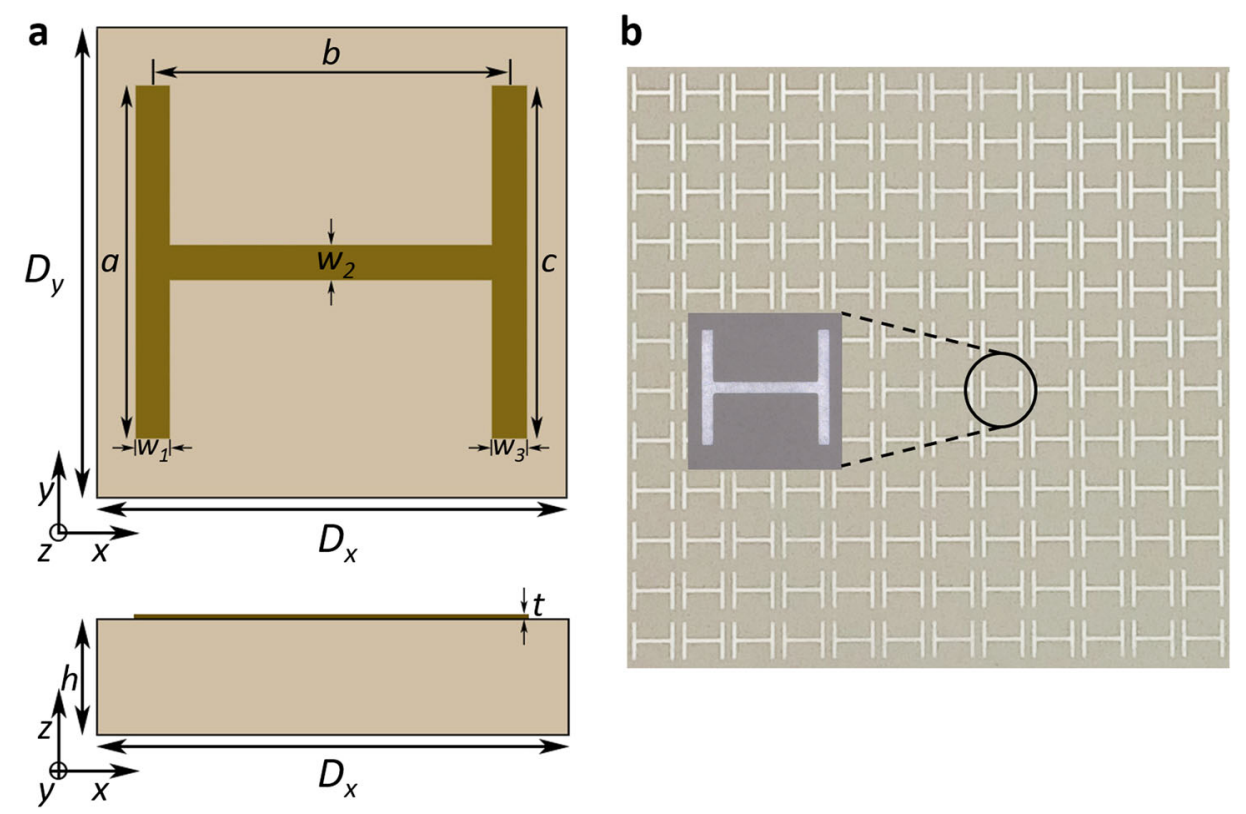

Fig. 1 Geometrical details. a Geometry of the designed metasurface with $D_{x}=D_{y}=5.0 \mathrm{~mm}, a=b=c=3.8 \mathrm{~mm}, w_{1}=w_{2}=w_{3}=0.4 \mathrm{~mm}$ and $t=35$ $\mu \mathrm{m}$. $\mathbf{b}$ Top-view depiction of a sample with inset showing the details of a single unit

Another remarkable resonance responsible for asymmetric spectra, known as Fano-resonance, is very famous in photonic systems and has led to numerous applications in electromagnetically induced transparency, slow light, lasing, switching and sensing based on the resulting sharp asymmetrical spectra ${ }^{24-29}$. In particular, high-ordered multipoles, e.g., electric quadrupoles, supported by subwavelength units of the metasurface usually appear as dark modes in contrast to the fundamental electric dipolar mode, which gives rise to the characteristic Fano line shape in the transmission/refection spectra. However, the effects of Fano-resonance on the modes of the metasurfaces bound below the light line have seldom been studied before.

Position sensors play indispensable roles in microfluidics and biology and in the touch screens that are now widely used in daily life. The working principles of recent commercial position sensors include four types: capacitive, resistive, surface acoustic waves (SAWs), and infrared $(\mathrm{IR})^{30-32}$. Their technological limitations include complicated calibration processes due to multilayer construction for capacitive and resistive position sensors, restrictions on device size and substrate materials (piezoelectrics) for SAW sensors, and low sensing resolution for IR sensors $^{30}$.

In this paper, we study the effects of Fano-resonance on the bound modes in metasurfaces and demonstrate that Fano-resonance can lead to hyperbolic IFCs, especially for lower Q-factor resonance, which acts analogously to symmetrical resonance. We designed a metasurface with
Lifshitz-like IFC transitions in different bands in two orthogonal directions corresponding to independent frequencies. Compared with previous work, the existence of independent collimation frequencies for orthogonal directions in our design shows great potential for applications not only in superlenses and wavefront control but also in position sensors ${ }^{19}$. Accordingly, we built a position-sensing prototype device to demonstrate that the designed metasurface can function as a position sensor by working in tandem with recognition systems. The prototype represents a new kind of position sensor based on surface EM waves that has never been reported before.

\section{Materials and methods}

We start with a unit cell composed of a dielectric slab and an anisotropic metallic pattern (H-shape), as shown in Fig. 1, in which the dielectric slab is made of a nonmagnetic material with a relative permittivity of 16 (with 0.001 tangential loss) and a thickness $h=1 \mathrm{~mm}$. The geometrical parameters of the metallic pattern are chosen as follows: period $D_{x}=D_{y}=5.0 \mathrm{~mm}, a=b=c=3.8 \mathrm{~mm}$, $w_{1}=w_{2}=w_{3}=0.4 \mathrm{~mm}$ and thickness $t=35 \mu \mathrm{m}$. Various EM properties, including SSPP propagation, reflection, and transmission, are strongly influenced both by these geometric parameters and by the relative permittivity of the dielectric slab and are well predicted by their band structures, especially for $2 \mathrm{D}$ materials ${ }^{22,33}$. Therefore, we first evaluated the band diagram of our designed metasurface, as shown in Fig. 2, where Fig. 2a represents the band structure below the light line (blue dash lines), and 

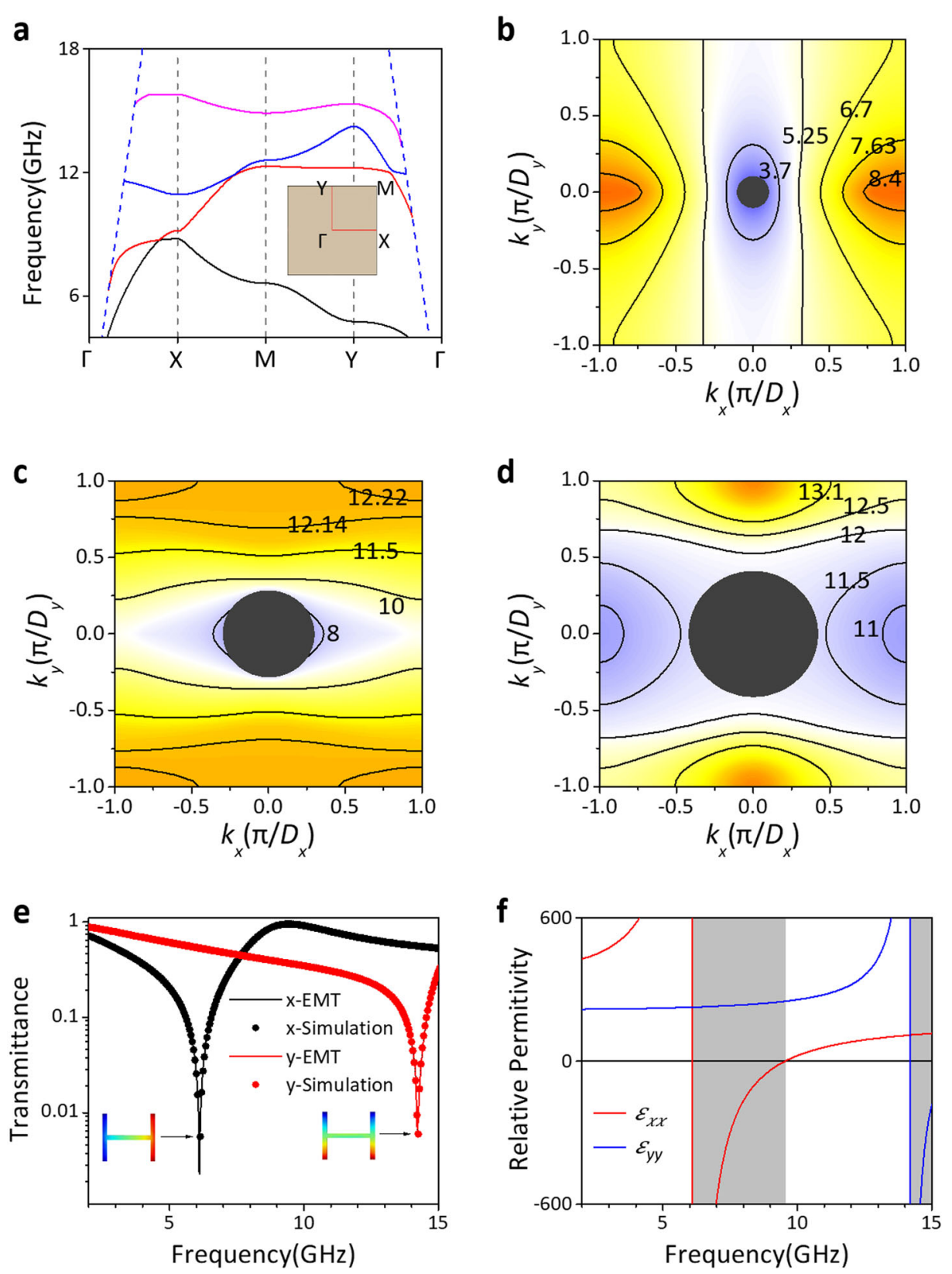

Fig. 2 Diagrams for $\boldsymbol{a}=\mathbf{3 . 8} \mathbf{~ m m}$. a Band diagram below light lines with frequency in GHz. The inset shows the Brillouin zone and high-symmetrical points. b-d IFCs of the first three bands in the first Brillouin, respectively, where the gray discs located at the center are the light cones at $3 \mathrm{GHz}, 9$ $\mathrm{GHz}$, and $12.5 \mathrm{GHz}$, respectively, the frequency values are marked in $\mathrm{GHz}$, and the frequency increases from blue to orange. e Transmission spectra at normal incidence obtained from simulations (EMT) when the electric field is polarized along the $x$ - and $y$-directions, corresponding to the black line (point) and the red line (point), respectively. Insets are the $E_{z}$ distribution at different resonances with values varying from -max (blue) to max (red). $\mathbf{f}$ Relative permittivity obtained from EMT, where the gray regions indicate the frequency ranges of hyperbolic IFCs predicted by EMT

the inset indicates the first Brillouin zone and highly symmetrical points.

Further evaluation of the IFCs of the first three bands in Fig. $2 \mathrm{~b}-\mathrm{d}$ has revealed a topological transition of IFCs under a continuous frequency in the first band, where the shape of the IFCs gradually transforms from a closed ellipse to an open hyperbola, becoming a flat line at the transition point. The group velocity of the SSPP modes is perpendicular to the IFCs, which indicates collimation at the transition frequency due to the flat line feature ${ }^{19}$. Here, we employ the effective medium theory (EMT) to qualitatively facilitate the design of functional metasurfaces.

In most practical cases, the EM response of anisotropic metamaterials can be characterized by the effective 

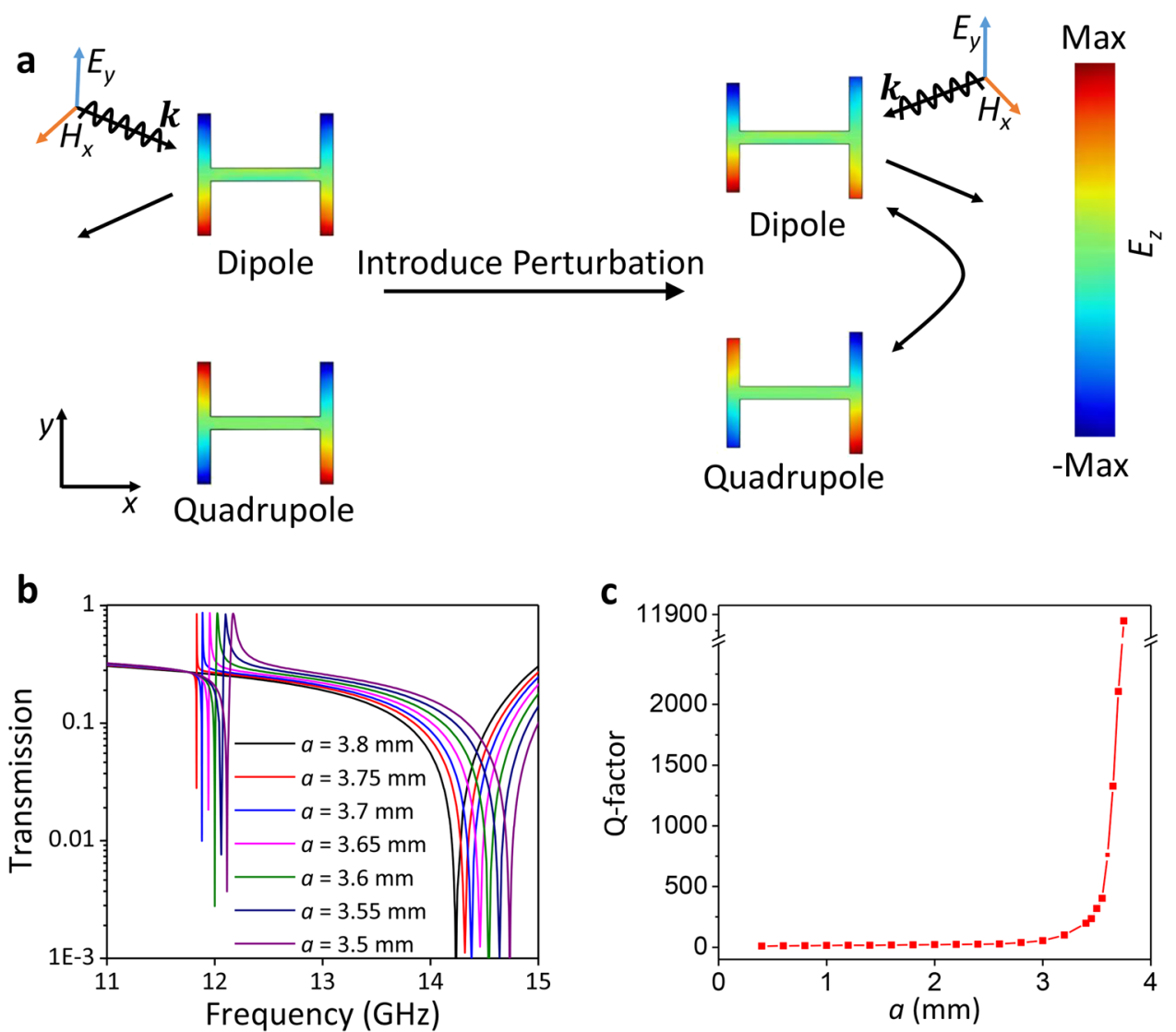

Fig. 3 The introduction of Fano-resonance. a Fano interference between the electric dipole and quadrupole modes at the left and right side, indicating the symmetric and asymmetric case, respectively. $\mathbf{b}$ Normal-incidence transmission spectra with different a when the electric field is polarized along the $y$-direction. c Variation in Q-factor obtained by fitting the Fano line shape as a function of (a)

permittivity and permeability tensors ${ }^{34-37}$. In our case, the permittivity $(\hat{\varepsilon})$ is anisotropic with orthogonal principle axes:

$$
\hat{\varepsilon}=\left(\begin{array}{cc}
\varepsilon_{x x} & 0 \\
0 & \varepsilon_{y y}
\end{array}\right),
$$

where the coordinates are normalized along the principle axis to diagonalize the matrix, and the subscripts $x x$ and $y y$ denote the $x$ and $y$-directions, respectively. Notably, only TE modes (electric field polarized along the in-plane directions) are included in the designed metasurfaces due to their special resonances (see details in Supp. Note 3), which are quite different from those of the anisotropic metasurfaces with a metallic substrate, as TM modes are significant in the second case ${ }^{19,38}$. Next, we simplify the metasurfaces as a truly two-dimensional system and set the wave vector along the $z$-direction equal to a constant as an approximation, and the shapes of the IFCs of the designed metasurfaces can then be predicted by ${ }^{39}$ :

$$
\frac{k_{x}^{2}}{\varepsilon_{y y}}+\frac{k_{y}^{2}}{\varepsilon_{x x}}=k_{0}^{2},
$$

where $k_{x}$ and $k_{y}$ are the propagation vectors in the $x$ and $y$-directions, respectively, and $k_{0}=\omega / c$ denotes the wavenumber of the substrate with angular frequency $\omega$ and light velocity in a vacuum $c$. The multipolar distributions in our designed metasurface are numerically studied (see Supp. Note 1), concentrating on the electric dipole mode. The effective permittivity along the $x$ and $y$ directions of the metasurface can be determined through fitting normal incident transmission spectra ${ }^{40,41}$. By employing Lorentzian dispersion and setting a thickness of $0.1 \mathrm{~mm}$ (see Supp. Note 2), the effective permittivity can be expressed as ${ }^{42}$ :

$$
\begin{array}{r}
\varepsilon_{x x}=163+\frac{8798.42}{6.1^{2}-f^{2}-0.032 i \cdot f} \\
\varepsilon_{y y}=175+\frac{8377.53}{14.12^{2}-f^{2}-0.082 i \cdot f},
\end{array}
$$

where the imaginary parts indicate the loss from the dielectric slab, since a 0.001 tangential loss has been set during simulations, and $f$ represents the frequency (in $\mathrm{GHz})^{43}$. The normal incident transmission spectra with 
the electric field polarized along the $x$ and $y$-directions are shown in Fig. 2d. Here, the red and black points denote the spectra from COMSOL Multiphysics simulations, while the solid lines indicate the homogenized approximation. The simulation and approximation are seen to be in good agreement. Equation (3) has been plotted in Fig. 2f, where the gray regions $(6.1-9.6 \mathrm{GHz}$ and $>14.12 \mathrm{GHz})$ predict hyperbolic IFCs and collimation at the transition frequency $(6.1 \mathrm{GHz})$. The band diagram from EMT has been studied (see Supp. Note 3), and the deviation can be understood by considering the strong overlap of frequencies between the different bands and the subwavelength-incompatible geometries at increased frequency, since EMT becomes invalid under such conditions. Therefore, the equation does successfully predict the existence of hyperbolic IFCs; that is, hyperbolic IFCs can be introduced by symmetrical resonance and have good accuracy when the structure is deeply subwavelength.

Unfortunately, the extensive overlap between the frequencies of the second and third bands indicates that there is no independent Lifshitz-like continuous topological transition in this frequency region, while the modes corresponding to the relatively flat IFCs in the second band, as shown in Fig. 2c are difficult to excite individually. There are two approaches to addressing this issue in order to obtain the other independent nondegenerate collimation frequency in the $y$-direction: one is to break the overlap of frequencies between the bands, and the other is to introduce a new resonance that can be excited by plane EMWs. First, we numerically calculate the modes at the resonance frequencies, as shown in the inset in Fig. 2e, where the mode at the higher frequency is a symmetrical dipole mode with dipole momentum along the $y$-direction. Consequently, there could be a dark mode protected by symmetry along the $y$-direction. As shown in Fig. 3a, when our designed $\mathrm{H}$-shape is symmetrical with respect to the $y$ direction, the electric quadrupole mode (EQM) fails to be excited by the incident EM wave because it is decoupled from the electric dipole mode; however, it is no longer a dark mode once the mirror symmetry along the $y$-direction is broken, as it will couple with the electric dipole mode in this case. Therefore, our strategy to create nondegenerate collimation along the $y$-direction is to develop Fanoresonance in our system, that is, to sabotage the $y$-directional symmetry of the metallic pattern. For simplicity, we decrease only the length of $a$ and keep the other parameters unaltered. The transmission coefficient for this asymmetrical structure when the electric field is polarized along the $y$-direction can be described by temporal coupled-mode theory $^{44}$ :

$$
t_{n}=1-\frac{\left|\alpha_{E}\right|^{2}}{\left(f-\vec{\omega}_{d}\right)-\frac{|\kappa|^{2}}{\left(f-\vec{\omega}_{q}\right)}},
$$

where $t_{n}$ denotes the normal incident transmission coefficient; $\alpha_{E}$ represents the radiative coupling parameter; $\vec{\omega}_{d}=\omega_{d}+i \alpha_{E}^{2}$ and $\vec{\omega}_{p}=\omega_{p}$, where $\omega_{d}$ and $\omega_{p}$ are the resonant frequencies corresponding to the dipole mode and quadrupole mode, respectively; and $\kappa$ is the coupling coefficient between these two modes (which is a function of the degree of asymmetry of the pattern) at normal incidence. It is equal to 0 when our structure is symmetrical, which indicates only one dip in the transmission spectrum due to dipole mode, whereas there are two dips for an asymmetric structure.

Here, $a=3.8 \mathrm{~mm}, a=3.75 \mathrm{~mm}, a=3.7 \mathrm{~mm}, a=3.65$ $\mathrm{mm}, a=3.6 \mathrm{~mm}, a=3.55 \mathrm{~mm}$ and $a=3.5 \mathrm{~mm}$ are used as the parameters for the normal incident transmission spectra with the electric field polarized along the $y$ direction. As shown in Fig. 3b, the asymmetric line shape (Fano line shape) with the symmetry broken along the $y$ direction has been directly observed. Notably, Equation (4) is not suitable for fitting the transmission in Fig. 3b, because the multipole modes have been defined according to the current contributions of the metallic pattern, in which the effects of the dielectric slab are not fully included, especially in the evaluation of transmission. Nevertheless, it is fruitful for the qualitative prediction of the dip corresponding to the quadrupole and dipole modes and is very correct once only metallic patterns are included (see Supp. Note 10). The Q-factor was evaluated by fitting the transmission spectra with the Fano line shape (see Supp. Note 4), which decreases exponentially as length $a$ decreases, as shown in Fig. 3c. The resonance frequency shows a blueshift, which is also studied (see Supp. Note 5). Recently, Fano-resonance with a high Qfactor has been used in many areas, such as chemical sensing ${ }^{45}$ and slow light ${ }^{27}$. However, it is arduous to establish an efficient form to describe the effective permittivity and permeability in this case, and resonances with high Q-factors, above the light line, will couple with modes below ${ }^{46}$. Therefore, resonances with a lower Qfactor were chosen for our purpose.

The Fano line shape with a low Q-factor behaves like a symmetrical one, in which the effective parameters can be described via Lorentzian expressions. Here, $a=2.6 \mathrm{~mm}$ is selected as an example since the Q-factor is $<100$, as shown in Fig. 3c. The contributions to the radiating power for the case of $a=2.6 \mathrm{~mm}$ are dominated by the EQMs, which has been numerically verified (see Supp. Note 1). The contributions of electric multipole modes (including EQMs) to the effective parameters in metamaterials after homogenization has been studied ${ }^{47,48}$. In particular, the EQMs in a metasurface contribute not only to the effective permittivity in the plane but also to the effective permeability perpendicular to the plane $\left(\mu_{z z}\right)$, which is related to the in-plane permittivity ${ }^{49,50}$. In addition, interestingly, the bianisotropic property predicts that the 

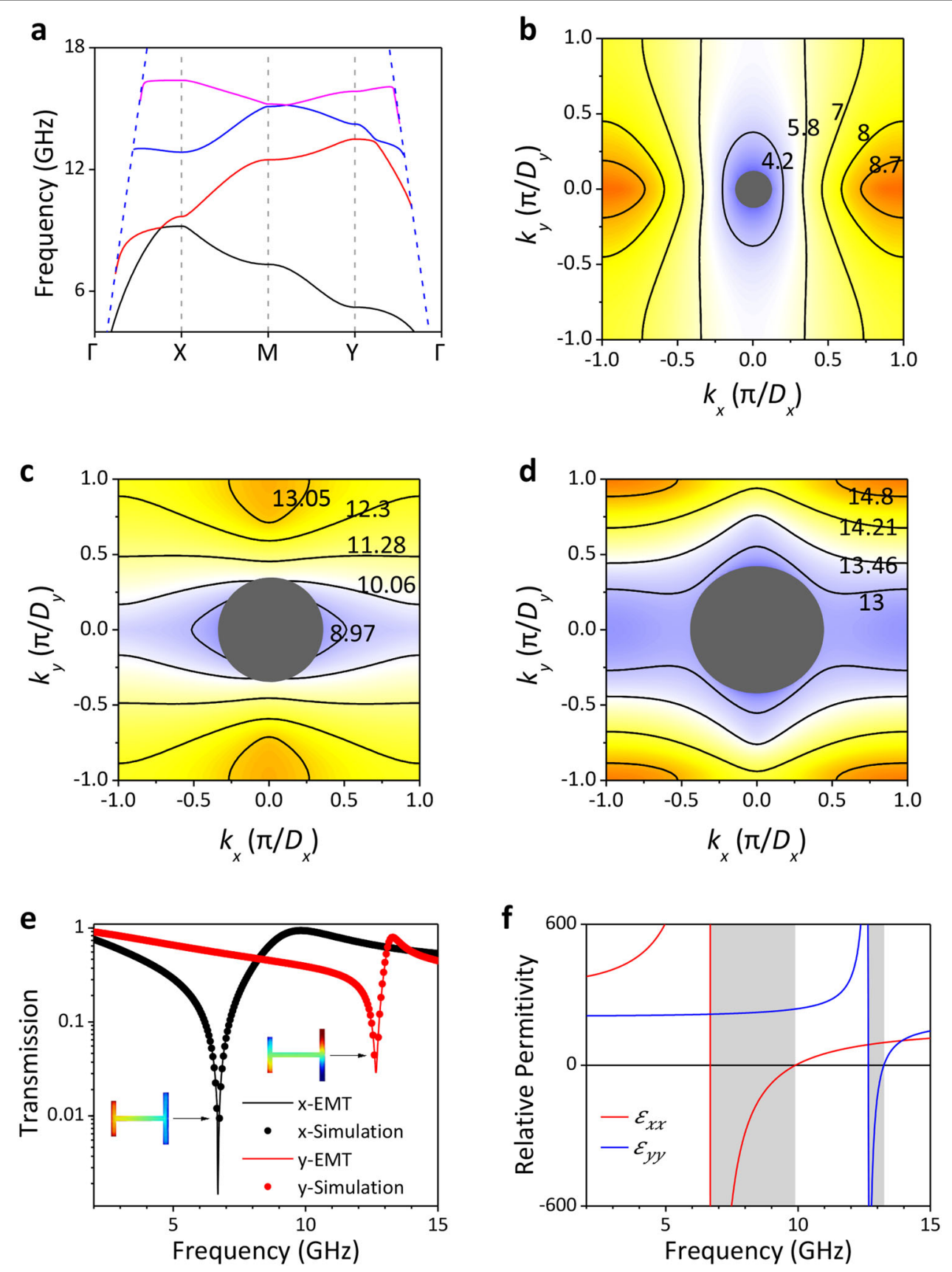

Fig. 4 Diagrams for $\boldsymbol{a}=\mathbf{2 . 6} \mathbf{~} \mathbf{~ m}$. a Band diagram below light lines with frequency in GHz. $\mathbf{b}-\mathbf{d}$ IFCs of the first three bands in the first Brillouin zone, where the gray discs located at the center are the light cones at $3.7 \mathrm{GHz}, 10.5 \mathrm{GHz}$ and $13.2 \mathrm{GHz}$, respectively. Frequency values are marked in $\mathrm{GHz}$, and the frequency increases from blue to orange. e Transmission spectra at normal incidence obtained from simulations (EMT) when the electric field is polarized along the $x$ and $y$-directions, corresponding to the black line (point) and the red line (point), respectively. Insets are the Ez distribution at different resonances with values varying from - max (blue) to max (red). $\mathbf{f}$ Effective parameters obtained from EMT, where the gray regions indicate the frequency regions of the hyperbolic IFCs predicted by EMT

corresponding resonant frequency for the in-plane permittivity exists when $\mu_{z z}$ vanishes, namely, that $\mu_{z z}$ has no contribution to determining the shape of IFCs in an application in our case ${ }^{49}$. We note that local constitutive parameters still make sense in the prediction of hyperbolic IFCs, even though the metasurface should be regarded as nonlocal once the EQMs are included. It is known that only in-plane effective parameters can be directly determined via normal transmission spectra, and therefore the effective permittivity can be fitted. With a 

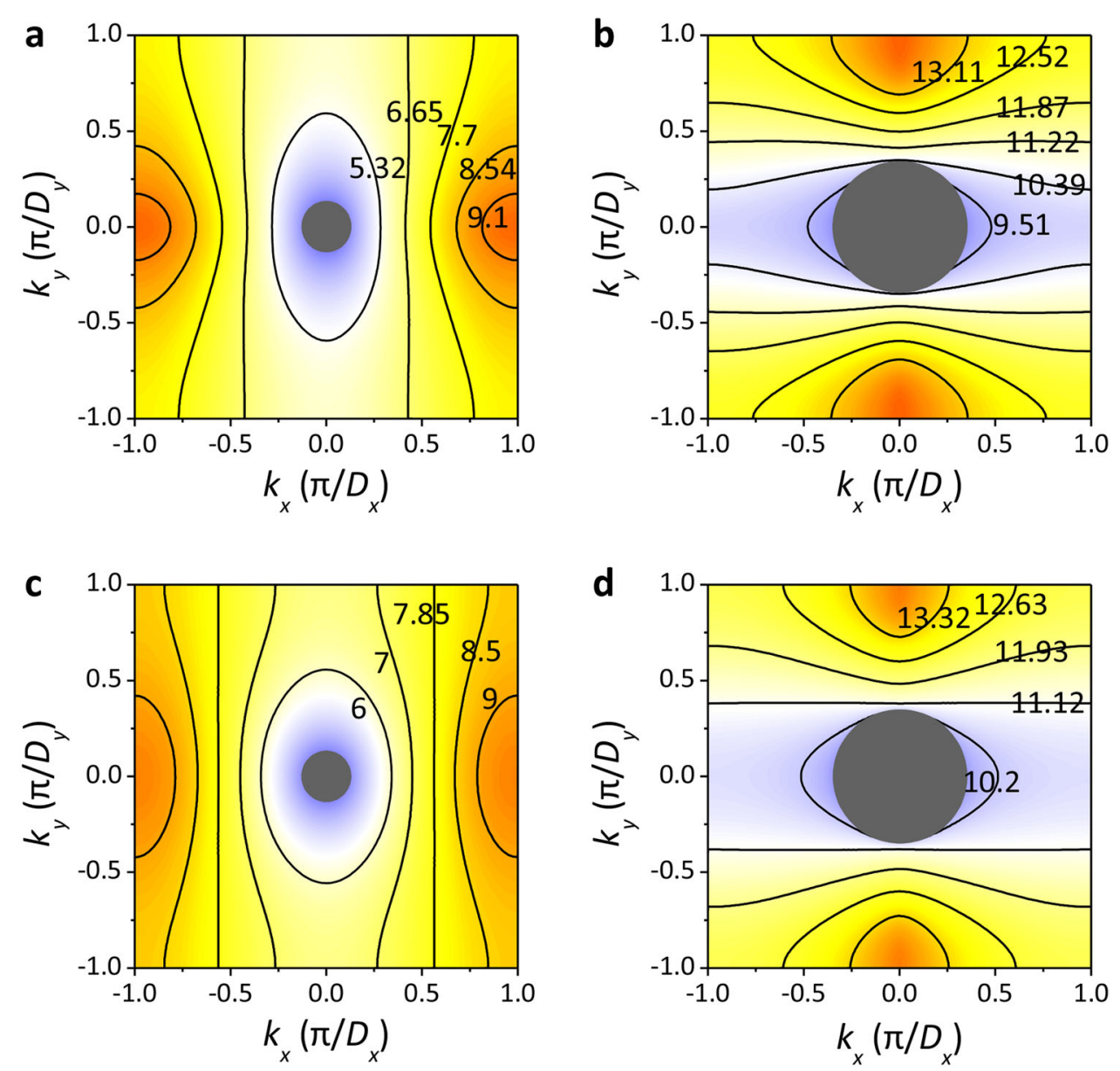

Fig. $\mathbf{5}$ IFCs of the first two bands. a, $\mathbf{c}$ The IFCs of the first bands for $a=1.4 \mathrm{~mm}$ and $a=0.4 \mathrm{~mm}$, respectively. $\mathbf{b}$, $\mathbf{d}$ The IFCs of the second bands for $a=1.4 \mathrm{~mm}$ and $a=0.4 \mathrm{~mm}$, respectively. The gray discs located at the center are the light cones at $3.7 \mathrm{GHz}$ for (a), (c) and at $10.8 \mathrm{GHz}$ for (b), (d), respectively. Frequency values are marked in $\mathrm{GHz}$, and the frequency increases from blue to orange

thickness of $0.1 \mathrm{~mm}$, the expressions read:

$$
\begin{array}{r}
\varepsilon_{x x}=163+\frac{8721.95}{6.68^{2}-f^{2}-0.024 i \cdot f} \\
\varepsilon_{y y}=175+\frac{2848.3}{10.65^{2}-f^{2}-0.09 i \cdot f} \\
+\frac{427.6}{17.1^{2}-f^{2}-0.2 i \cdot f}
\end{array}
$$

Here, the effect of Fano-resonance on $\varepsilon_{x x}$ is neglected because the current distribution is concentrated in the $y$ direction, which has been numerically verified (see Supp. Note 6).The transmission spectra and effective parameters are shown in Fig. 4e, f, respectively, indicating two hyperbolic IFC regions marked in gray, where $\varepsilon_{x x}$ and $\varepsilon_{y y}$ have opposite signs. To verify our deduction, the IFCs were numerically evaluated, as shown in Fig. $4 \mathrm{~b}-\mathrm{d}$. A Lifshitz-like topological transition of the IFCs occurs in the second band with a flat line at the transition frequency, which indicates the other collimation frequency with group velocity along the y-direction. The Lifshitz-like transition of the IFCs for the first band is always retained, as the physical conditions are consistent, and the collimation is very robust as $a$ is decreased. We note that the same phenomenon still occurs even when $a$ has been reduced to $0.4 \mathrm{~mm}$, and thus, the metallic pattern can be regarded as a 'T-shape'. The IFCs of the first two bands for $a=1.4 \mathrm{~mm}$ and $a=0.4 \mathrm{~mm}$ have been plotted in Fig. 5.

To summarize our design, we successfully created a continuous Lifshitz-like topological transition for the IFCs in the $y$-direction by introducing low-Q Fano-resonance to our system. Accordingly, by designing continuous nondegenerate topological transitions in orthogonal directions, two flat IFCs with independent frequencies in orthogonal directions can be achieved. This phenomenon is very stable under variable geometries. Very recently, the modes corresponding to the transition frequency in a single direction 
a

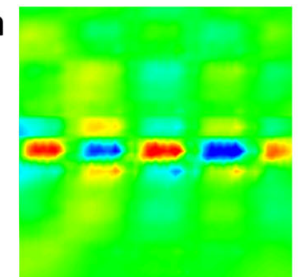

@ $5.29 \mathrm{GHz}$

e

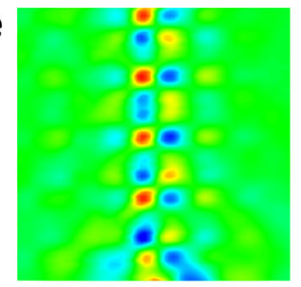

@ $11.63 \mathrm{GHz}$

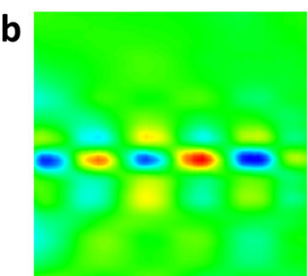

@ $6.04 \mathrm{GHz}$

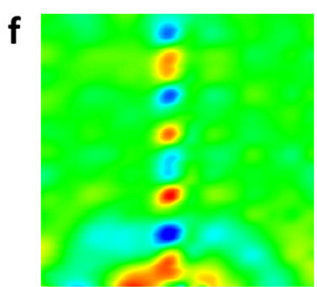

@ $11.51 \mathrm{GHz}$

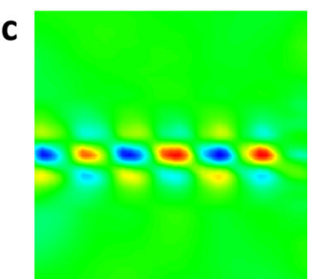

@ $6.9 \mathrm{GHz}$

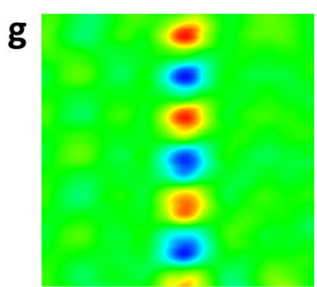

@ $11.4 \mathrm{GHz}$

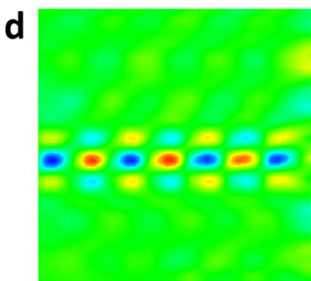

@ $7.9 \mathrm{GHz}$

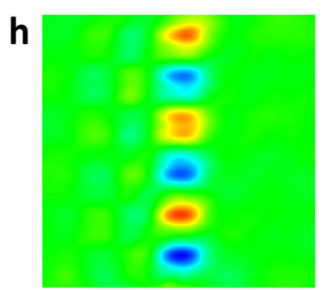

@ $11.2 \mathrm{GHz}$

Fig. 6 Measured field distributions. a-d $E_{x}$ distribution when the metasurface is excited along the $x$-direction, for $a=3.8 \mathrm{~mm}, 2.6 \mathrm{~mm}, 1.4 \mathrm{~mm}$, and $0.4 \mathrm{~mm}$, respectively. $\mathbf{e}-\mathbf{h} E_{x}$ distribution when excited along the $y$-direction, with $a=3.8 \mathrm{~mm}, 2.6 \mathrm{~mm}, 1.4 \mathrm{~mm}$, and $0.4 \mathrm{~mm}$, respectively

were proven to be diffractionless, soliton-like and sensitive to the surrounding environment ${ }^{51}$. To the best of our knowledge, this study is the first time that collimation has been demonstrated in both orthogonal directions at independent frequencies, which enables many potential applications, especially for position sensors. Meanwhile, recent work on Fano-resonance has mainly concentrated on transmission and reflection spectra, whereas we highlight that Fano-resonances are also accountable for the control of surface modes located below the light line, which introduces a new and previously unreported avenue for manipulating more complicated bands of metasurfaces ${ }^{52-55}$. In the next section, we describe the microwave experiment and a sensing prototype, further confirming our results.

\section{Results}

\section{Experiment}

A metasurface consisting of $14 \times 14$ unit cells and excitation units (total size $8 \times 8 \mathrm{~cm}^{2}$ ) was fabricated. The geometric parameters of the unit cell were chosen as in Fig. 1a, and the excitation unit was composed of microstrip lines to excite surface EM waves (see Supp. Note 7). The details of our real sample are shown in Fig. 1b. The dielectric slab is made of TP1/2, a nonmagnetic material with a relative permittivity of 16 and a tangential loss of 0.001 . The metallic patterns were printed in the dielectric slab with $35 \mu \mathrm{m}$ thick copper and were covered by a negligible thickness of solder on the copper surface to prevent oxidation.

The symmetrical case $(a=3.8 \mathrm{~mm})$ was studied first. Figure 6a shows the distribution of the electric field along the $x$-direction $\left(E_{x}\right)$, which is measured by locating a receiving antenna $\sim 1 \mathrm{~mm}$ above the metasurface when the input frequency is $5.29 \mathrm{GHz}$ along the $x$-direction.
Compared with the IFCs in Fig. 2b, the frequency for the flat IFC agrees well with our simulation results, and the slight difference between the experiment and the simulation is due to the fabrication tolerances and the derivation of relative permittivity. However, we fail to find a collimation frequency upon excitation along the $y$-direction, and the $E_{x}$ distribution for a relatively unidirectional propagation frequency $(11.63 \mathrm{GHz})$ in our experiment is plotted in Fig. 6e. The modes composed of $x$ components have been excited, and the $E_{x}$ distribution is observed in a large area instead of along a narrow trail. Compared with Fig. 2c, d, it is evident that modes in the third band at the same frequency have also been excited, resulting in group velocity along the $x$-direction.

Next, the asymmetric cases were studied. Figure $6 \mathrm{~b}, \mathrm{f}$ depicts the $E_{x}$ distribution in the case of $a=2.6 \mathrm{~mm}$ when the input frequency is $6.04 \mathrm{GHz}$ along the $x$-direction and $11.51 \mathrm{GHz}$ along the $y$-direction. Collimation is maintained along the $\mathrm{x}$-direction, which is similar to the symmetric case. However, in contrast to Fig. 6e, mode propagation along a narrow region can be observed in Fig. 6f, which is consistent with the prediction of the IFCs in Fig. 4, where an independent flat line exists for the second band. The cases of $a=1.4 \mathrm{~mm}$ and $a=0.4 \mathrm{~mm}$ were also studied, where Fig. 6c, g corresponds to $a=1.4$ $\mathrm{mm}$ and Fig. $6 \mathrm{~d}$, h to $a=0.4 \mathrm{~mm}$, respectively. Therefore, two independent collimation frequencies along orthogonal directions in our designed metasurface have been found, proving that hyperbolic IFCs can be introduced by low-Q Fano-resonance. The $E_{x}$ distributions corresponding to the collimation frequencies were also studied via COMSOL Multiphysics (see Supp. Note 8). The collimation modes are sensitive to the surrounding environment, and the electric field is well confined in a narrow 

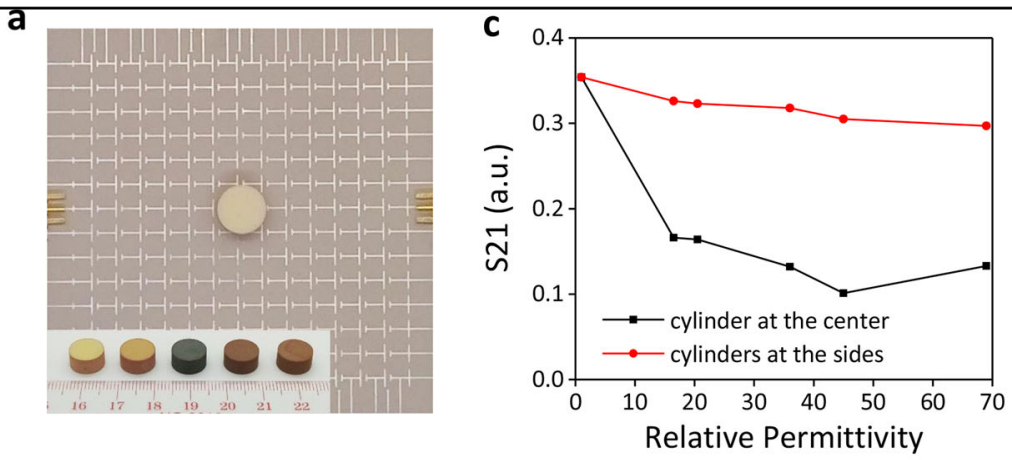

b
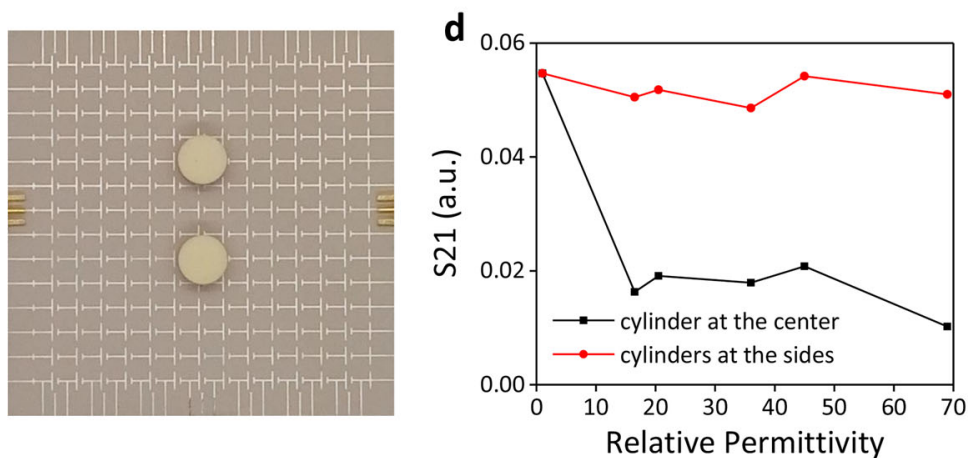
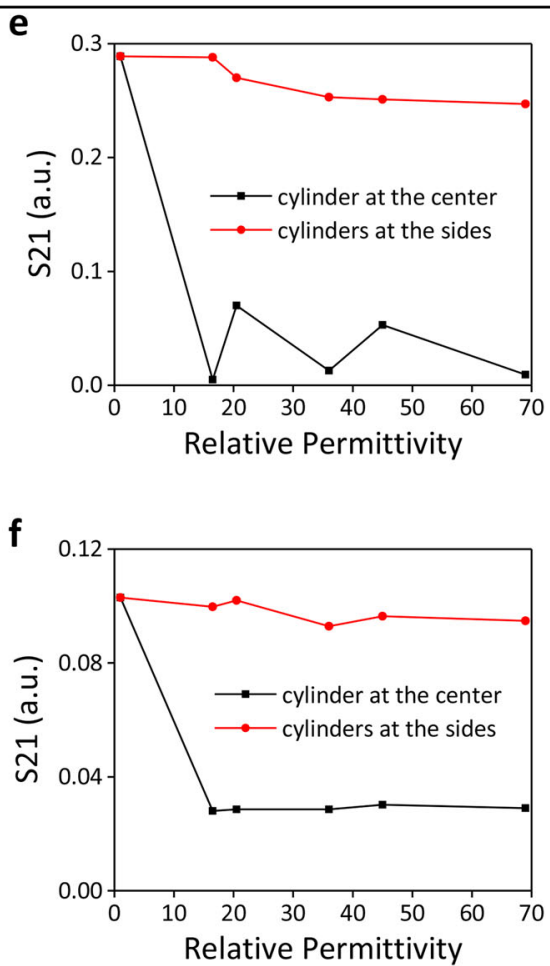

Fig. 7 The effects of the perturbation. $\mathbf{a}, \mathbf{b}$ Schematics with cylinders placed at the center and at the sides of the propagation path, respectively. The inset in (a) shows cylinders with relative permittivities of $16.5,20.5,36,45$, and 69 from left to right. c-f S21 amplitudes of (c) $a=1.4$ mm, field along the $x$-direction; (d) $a=1.4 \mathrm{~mm}$, field along the $y$-direction; (e) $a=0.4 \mathrm{~mm}$, field along the $x$-direction; and (f) $a=0.4 \mathrm{~mm}$, field along the $y$ direction

region at the collimation frequency. Based on the physical features, we performed the following experiment to demonstrate that collimation modes can be used to read minor changes in the environment, which is the foundation of position sensors.

To change the environment quantitatively, dielectric cylinders with both radius and height equal to $5 \mathrm{~mm}$ were employed, as shown in the inset in Fig. 7a (the dielectric cylinders have relative permittivities of 16.5 , 20.5, 36, 45 and 69 from left to right). Here, the metasurface of $a=1.4 \mathrm{~mm}$ with excitation along the $x$-direction is shown in Fig. 7a, b (the former has the cylinder placed at the center of the propagation trail, and the latter has cylinders placed at the sides of the trail). The S21 amplitude was measured for $6.9 \mathrm{GHz}$ along the $x$ direction and $11.4 \mathrm{GHz}$ along the $y$-direction, as shown in Fig. 7c, d, respectively, in the case of $a=1.4 \mathrm{~mm}$. For $a=0.4 \mathrm{~mm}$, the cases with $7.9 \mathrm{GHz}$ along the $x$-direction and $11.2 \mathrm{GHz}$ along the $y$-direction are plotted in Fig. 7e, f, respectively. The S21 amplitude was considerably reduced when a dielectric cylinder was placed on the propagation trail and fluctuated slightly in the off-trail case, which reveals that interference from the environment of the propagation trail can be registered via S21 amplitude.

\section{Position-sensing prototype}

The prototype device is composed of two independent microwave sources, four microwave switches, two amplifiers, two detectors, a voltage collector, some coaxial cables and an analysis system, as shown in Fig. 8. The switches are controlled by the program, allowing the ports with the numbers marked on the sample to be periodically opened or closed. For instance, suppose $T$ is the opening time of each switch, and only the four ports labeled 1 are open in the time period $0-T$, only the four ports labeled 2 are open in the time period $T-2 T$, and so on. Then, EM waves emitted from the microwave source can periodically scan from port 1 to port 5 with a periodicity of $5 T$. The EM waves received from switch 2 and switch 4 are amplified because of the limited coupling efficiency from the plane EM waves to the surface EM waves, and there is substantial loss when transmitting though coaxial cables and switches, so that the power of the EM waves can decay to less than the detection limit of the detectors. The EM wave signal is converted into an AC voltage signal upon reaching the detector after amplification and is collected by the voltage collector. As a result, each row and column is marked by independent numbers, and 25 total points can be identified and distinguished via the recognition system when the amplitude of the signal is decreased by 


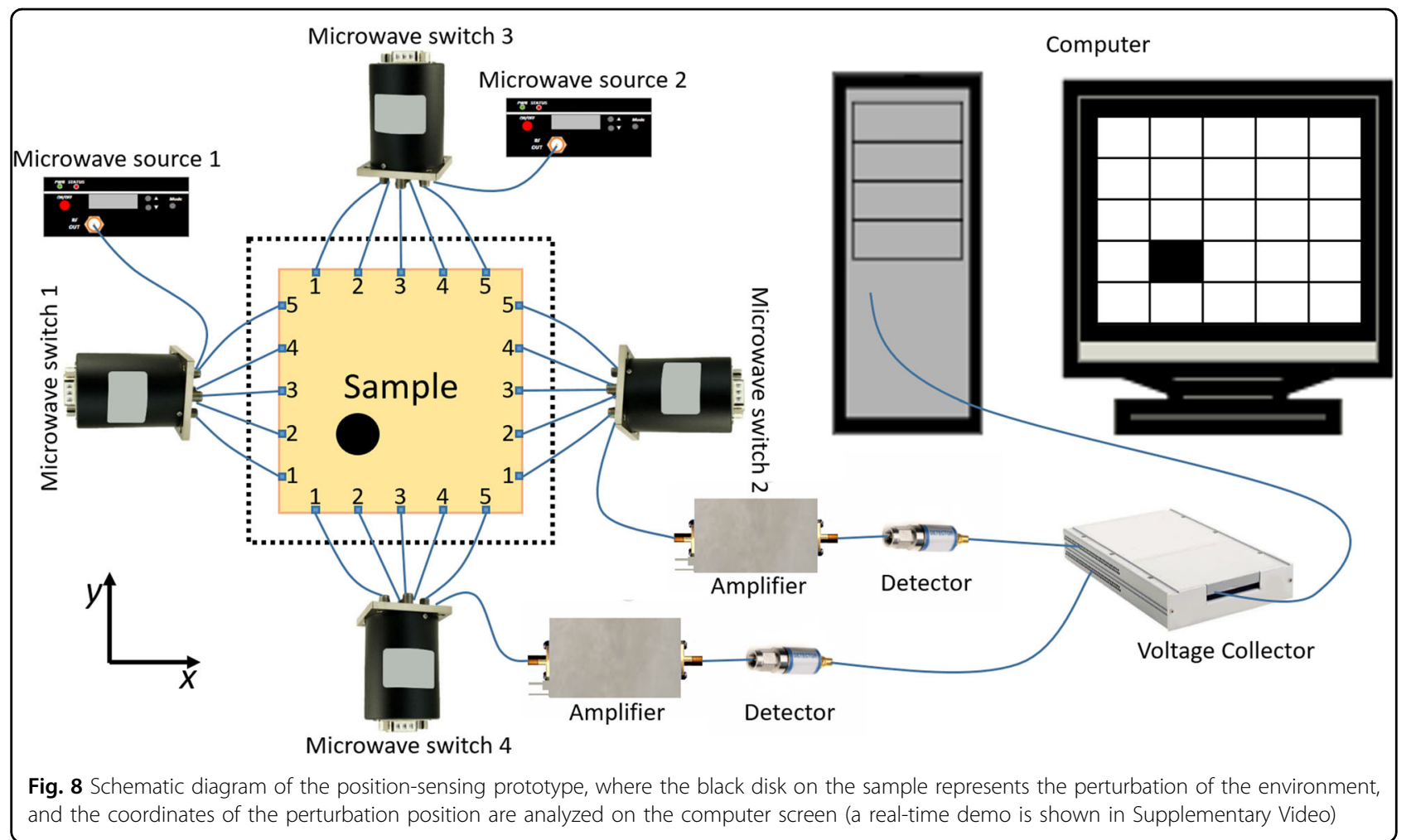

the touch of a finger on the surface. In our recognition system, touch location is mapped on the screen and refreshed each period.

Since the collimations are very stable under a constant physical state, it is feasible to adjust the geometrical parameters to $D_{x}=D_{y}=6.0 \mathrm{~mm}, a=3 \mathrm{~mm}, b=c=5$ $\mathrm{mm}, w_{1}=w_{2}=w_{3}=0.4 \mathrm{~mm} d=1 \mathrm{~mm}$ and $t=35 \mu \mathrm{m}$ to decrease the collimation frequency along the $x$-direction due to the limitations of our microwave source. The IFCs of the first two bands under this geometry have been evaluated and plotted (see Supp. Note 9). The real sample is composed of $20 \times 20$ units, and the excitations were kept unchanged. Two independent collimation frequencies were identified, equal to $4.78 \mathrm{GHz}$ and $9.5 \mathrm{GHz}$ in the $x$ and $y$-directions, respectively (see Supp. Note 9). The open time $T$ is set to $0.6 \mathrm{~s}$ due to the limitations of the switching time of our microwave switch, whose periodicity is $3 \mathrm{~s}$.

In Fig. 8, the black disk denotes the perturbation of the environment at the touch of a finger, as shown in the video (see Supp. Video). The coordinates of the position we touched were successfully mapped on the screen, which establishes that the metasurface based on the given design can function as a position sensor. Other properties, including the touch resolution and the loss of the designed position sensor, have also been discussed (see Supp. Note 9). To the best of our knowledge, this is the first realization of a position sensor based on surface EM waves. Many advantages can be found compared to current position sensors; for instance, this sensor has simpler layers and more easily adjusted resolution than capacitive and resistive sensors and broader optional substrates and wider application ranges than surface acoustic wave sensors $^{30-32}$.

\section{Discussion}

In conclusion, we have shown that the Fano-resonance (especially with a lower Q-factor) behaves like a symmetrical resonance and can give rise to hyperbolic IFCs in both EMT and numerical simulation. Then, we created topological transitions of IFCs in orthogonal directions in different bands by decreasing the Q-factor of the Fanoresonance in our system. Thus, the collimating phenomena in both orthogonal directions at the transition frequency were verified both numerically and experimentally. Moreover, a position sensor based on surface EM waves was prototyped and demonstrated. This demonstration was enabled by the fact that the collimated surface modes have great sensitivity to environmental changes in the propagation trails, and it illustrates one of several promising applications.

\section{Acknowledgements}

C.H. would like to thank Prof. C T Chan, Dr. Anan Fang, and Dr. Xiao Xiao for their helpful discussions. The work is supported by an Areas of Excellence Scheme grant (AOE/P-02/12) from the Research Grant Council (RGC) of Hong Kong, the Special Fund for Agro-scientific Research in the Public Interest from 
the Ministry of Agriculture of the Peoples' Republic of China (No. 201303045), and grants from the National Natural Science Foundation of China (NSFC) (No. 11474212) and the Priority Academic Program Development (PAPD) of Jiangsu Higher Education Institutions.

\section{Author details}

'Department of Physics, The Hong Kong University of Science and Technology, Clear Water Bay, Kowloon 518000 Hong Kong, China. ${ }^{2}$ Department of Applied Physics, Chongqing University, Chongqing 401331, China. ${ }^{3}$ College of Physics, Optoelectronics and Energy \& Collaborative Innovation Center of Suzhou Nano Science and Technology, Soochow University, Suzhou 215006, China. ${ }^{4}$ Key Laboratory of Modern Optical Technologies of Ministry of Education \& Key Laboratory of Advanced Optical Manufacturing Technologies of Jiangsu Province, Suzhou 215006, China

\section{Conflict of interest}

The authors declare that they have no conflict of interest.

\section{Publisher's note}

Springer Nature remains neutral with regard to jurisdictional claims in published maps and institutional affiliations.

Supplementary information is available for this paper at https://doi.org/ 10.1038/s41427-018-0047-0.

Received: 20 November 2017 Revised: 01 March 2018 Accepted: 27 March 2018.

Published online: 24 May 2018

\section{References}

1. Sun, S. et al. Gradient-index meta-surfaces as a bridge linking propagating waves and surface waves. Nat. Mater. 11, 426-431 (2012).

2. Polo, J. A. \& Lakhtakia, A. Surface electromagnetic waves: a review. Laser Photonics Rev. 5, 234-246 (2011)

3. Takayama, O., Crasovan, L., Artigas, D. \& Torner, L. Observation of Dyakonov surface waves. Phys. Rev. Lett. 102, 043903 (2009).

4. Homola, J. Surface plasmon resonance sensors for detection of chemical and biological species. Chem. Rev. 108, 462-493 (2008).

5. Fang, N., Lee, H., Sun, C. \& Zhang, X. Sub-diffraction-limited optical imaging with a silver superlens. Science 308, 534-537 (2005).

6. Zhang, X. \& Liu, Z. Superlenses to overcome the diffraction limit. Nat. Mater. 7, 435 (2008).

7. Gregoire, D. J. \& Kabakian, A. V. Surface-wave waveguides. IEEE Antennas Wirel. Propag. Lett. 10, 1512-1515 (2011).

8. Goubau, G. Surface waves and their application to transmission lines. J. Appl. Phys. 21, 1119-1128 (1950).

9. Wang, K. \& Mittleman, D. M. Metal wires for terahertz wave guiding. Nature 432, 376-379 (2004)

10. Pendry, J., Martin-Moreno, L. \& Garcia-Vidal, F. Mimicking surface plasmons with structured surfaces. Science 305, 847-848 (2004).

11. Liu, Y. \& Zhang, X. Metasurfaces for manipulating surface plasmons. Appl. Phys. Lett. 103, 141101 (2013)

12. Maguid, E. et al. Photonic spin-controlled multifunctional shared-aperture antenna array. Science 352, 1202-1206 (2016).

13. Liu, Z. et al. Hyper-interface, the bridge between radiative wave and evanescent wave. Appl. Phys. Lett. 96, 113507 (2010).

14. Glybovski, S. B., Tretyakov, S. A., Belov, P. A., Kivshar, Y. S. \& Simovski, C. R. Metasurfaces: from microwaves to visible. Phys. Rep. 634, 1-72 (2016).

15. Poddubny, A., lorsh, I., Belov, P. \& Kivshar, Y. Hyperbolic metamaterials. Nat. Photonics 7, 948 (2013).

16. Gao, W. et al. Topological photonic phase in chiral hyperbolic metamaterials. Phys. Rev. Lett. 114, 037402 (2015).

17. Xiao, M., Lin, Q. \& Fan, S. Hyperbolic Weyl point in reciprocal chiral metamaterials. Phys. Rev. Lett. 117, 057401 (2016).

18. High, A. A. et al. Visible-frequency hyperbolic metasurface. Nature 522, 192 (2015).

19. Yang, Y. et al. Hyperbolic spoof plasmonic metasurfaces. NPG Asia Mater. 9, e428 (2017).
20. Gomez-Diaz, J. S., Tymchenko, M. \& Alù, A. Hyperbolic plasmons and topological transitions over uniaxial metasurfaces. Phys. Rev. Lett. 114, 233901 (2015).

21. Witzens, J., Loncar, M. \& Scherer, A. Self-collimation in planar photonic crystals, IEEE J. Sel. Top. Quant. Elect. 8, 1246-1257 (2002).

22. Yermakov, Y. et al. Spin control of light with hyperbolic metasurfaces. Phys. Rev. B 94, 075446 (2016)

23. Yermakov, O. et al. Hybrid waves localized at hyperbolic metasurfaces. Phys. Rev. B 91, 235423 (2015)

24. Limonov, M. F., Rybin, M. V., Poddubny, A. N. \& Kivshar, Y. S. Fano resonances in photonics. Nat. Photonics 11, 543 (2017).

25. Zhang, S., Genov, D. A., Wang, Y., Liu, M. \& Zhang, X. Plasmon-induced transparency in metamaterials. Phys. Rev. Lett. 101, 047401 (2008).

26. Tassin, P., Zhang, L., Koschny, T., Economou, E. \& Soukoulis, C. M. Low-loss metamaterials based on classical electromagnetically induced transparency. Phys. Rev. Lett. 102, 053901 (2009).

27. Wu, C., Khanikaev, A. B. \& Shvets, G. Broadband slow light metamaterial based on a double-continuum Fano resonance. Phys. Rev. Lett. 106, 107403 (2011).

28. Amin, M., Ramzan, R. \& Siddiqui, O. Fano resonance based ultra high-contrast electromagnetic switch. Appl. Phys. Lett. 110, 181904 (2017).

29. Singh, R. et al. Ultrasensitive terahertz sensing with high-Q Fano resonances in metasurfaces. Appl. Phys. Lett. 105, 171101 (2014).

30. Cohen, N. Timeline: a history of touch-screen technology. National Public Radio 26 Dec. (2011).

31. Reis, S. et al. in IEEE International Symposium on Industrial Electronics (ISIE) 516-520 (IEEE, 2010).

32. Liu, Y., Nikolovski, J.P., Mechbal, N., Hafez, M. \& Vergé, M. An acoustic multitouch sensing method using amplitude disturbed ultrasonic wave diffraction patterns. Sens. Actuators A: Phys. 162, 394-399 (2010).

33. Patel, A. M. \& Grbic, A. Transformation electromagnetics devices based on printed-circuit tensor impedance surfaces. IEEE Trans. Microw. Theory Tech. $\mathbf{6 2}$ 1102-1111 (2014).

34. Arango, F. B. \& Koenderink, A. F. Polarizability tensor retrieval for magnetic and plasmonic antenna design. New J. Phys. 15, 073023 (2013).

35. Sun, L., Yang, X. \& Gao, J. Analysis of nonlocal effective permittivity and permeability in symmetric metal-dielectric multilayer metamaterials. J. Opt. 18, 065101 (2016).

36. Drachev, V. P., Podolskiy, V. A. \& Kildishev, A. V. Hyperbolic metamaterials: new physics behind a classical problem. Opt. Express 21, 15048-15064 (2013).

37. Shao, Z. et al. Manipulating surface plasmon polaritons with infinitely anisotropic metamaterials. Opt. Express 25, 10515-10526 (2017).

38. Hao, J. et al. Manipulating electromagnetic wave polarizations by anisotropic metamaterials. Phys. Rev. Lett. 99, 063908 (2007).

39. Chshelokova, A. V. et al. Hyperbolic transmission-line metamaterials. J. Appl. Phys. 112, 073116 (2012).

40. Koschny, T., Markoš, P., Smith, D. \& Soukoulis, C. Resonant and antiresonant frequency dependence of the effective parameters of metamaterials. Phys. Rev. E 68, 065602 (2003).

41. Smith, D., Schultz, S., Markoš, P. \& Soukoulis, C. Determination of effective permittivity and permeability of metamaterials from reflection and transmission coefficients. Phys. Rev. B 65, 195104 (2002).

42. Shen, J.T., Catrysse, P. B. \& Fan, S. Mechanism for designing metallic metamaterials with a high index of refraction. Phys. Rev. Lett. 94, 197401 (2005).

43. Hou, B., Xie, H., Wen, W. \& Sheng, P. Three-dimensional metallic fractals and their photonic crystal characteristics. Phys. Rev. B 77, 125113 (2008).

44. Mousavi, S. H., Khanikaev, A. B., Allen, J., Allen, M. \& Shvets, G. Gyromagnetically induced transparency of metasurfaces. Phys. Rev. Lett. 112, 117402 (2014).

45. Khanikaev, A. B., Wu, C. \& Shvets, G. Fano-resonant metamaterials and their applications. Nanophotonics 2, 247-264 (2013).

46. Lunnemann, P. \& Koenderink, A. F. The local density of optical states of a metasurface. Sci. Rep. 6, 20655 (2016).

47. Alù, A. First-principles homogenization theory for periodic metamaterials. Phys. Rev. B 84, 075153 (2011).

48. Silveirinha, M. G. Time domain homogenization of metamaterials. Phys. Rev. B 83, 165104 (2011)

49. Costa, J. T., Silveirinha, M. G. \& Maslovski, S. I. Finite-difference frequencydomain method for the extraction of effective parameters of metamaterials. Phys. Rev. B 80, 235124 (2009).

50. Silveirinha, M. G. Metamaterial homogenization approach with application to the characterization of microstructured composites with negative parameters. Phys. Rev. B 75, 115104 (2007). 
51. Gao, W.-L., Fang, F.-Z., Liu, Y.-M. \& Zhang, S. Chiral surface waves supported by biaxial hyperbolic metamaterials. Light.: Sci. Appl. 4, e328 (2015).

52. Yang, Y., Kravchenko, I. I., Briggs, D. P., \& Valentine, J. All-dielectric metasurface analogue of electromagnetically induced transparency. Nat. Commun. 5, 5753 (2014).

53. Mousavi, S. H. et al. Inductive tuning of Fano-resonant metasurfaces using plasmonic response of graphene in the mid-infrared. Nano Lett. 13, 1111-1117 (2013)
54. Dabidian, N. et al. Electrical switching of infrared light using graphene integration with plasmonic Fano resonant metasurfaces. ACS Photonics 2, 216-227 (2015).

55. Mousavi, S. H., Khanikaev, A. B. \& Shvets, G. Optical properties of Fano-resonant metallic metasurfaces on a substrate. Phys. Rev. B 85, 155429 (2012). 